\title{
A Sampling-based Boundary Detection Method with Consideration of Boundary Shape in Dense Mobile Wireless Sensor Networks
}

\author{
Kazuya Matsuo \\ Dept. of Multimedia \\ Engineering Graduate School \\ of Information Science and \\ Technology, Osaka Univ. \\ u.ac.jp \\ Akimitsu Kanzaki \\ Interdisciplinary Graduate \\ School of Science and \\ Engineering, Shimane Univ. \\ kanzaki@cis.shimane- \\ u.ac.jp
}

matsuo.kazuya@ist.osaka-goto.keisuke.osaka@gmail.com

\begin{abstract}
In this paper, we propose a method which efficiently detects the geographical boundaries of sensor readings in mobile wireless sensor networks, in situations where sensor data must be gathered within a short period. Typically, it is enough for detecting the boundaries to gather sensor data only from nodes near the boundaries. However, when the number of such nodes is large, it becomes impossible to gather sensor data from all these nodes within a short period, due to the frequent occurrence of packet collisions. To solve this problem, our proposed method reduces the number of sensor data to be gathered, by taking samples of nodes. When taking samples, our proposed method considers the target boundary shape, in order to suppress the deterioration in the accuracy of the boundaries estimated from the gathered sensor data.
\end{abstract}

\section{CCS Concepts}

-Networks $\rightarrow$ Network protocol design; Sensor networks; Mobile ad hoc networks;

\section{Keywords}

dense mobile wireless sensor networks; boundary detection; traffic reduction

\section{INTRODUCTION}

Permission to make digital or hard copies of all or part of this work for personal or classroom use is granted without fee provided that copies are not made or distributed for profit or commercial advantage and that copies bear this notice and the full citation on the first page. Copyrights for components of this work owned by others than ACM must be honored. Abstracting with credit is permitted. To copy otherwise, or republish, to post on servers or to redistribute to lists, requires prior specific permission and/or a fee. Request permissions from permissions@ acm.org.

MoMM '16, November 28-30, 2016, Singapore, Singapore

(c) 2016 ACM. ISBN 978-1-4503-4806-5/16/11 . \$15.00

DOI: http://dx.doi.org/10.1145/3007120.3007126

\author{
Keisuke Goto \\ Dept. of Multimedia \\ Engineering Graduate School \\ of Information Science and \\ Technology, Osaka Univ. \\ Takahiro Hara \\ Dept. of Multimedia \\ Engineering Graduate School \\ of Information Science and \\ Technology, Osaka Univ. \\ hara@ist.osaka-u.ac.jp
}

Recently, participatory sensing, wherein readings of sensed physical phenomena (sensor readings) and location information are gathered from portable sensor devices such as smart phones, has attracted much attention $[4,11]$. In participatory sensing, participants typically upload their acquired sensor data to the Internet through infrastructures such as $4 \mathrm{G}$ cellular networks. However, these networks are shared by many applications, and the data traffic generated by those applications has been growing every year [5], though the channel bandwidth of the networks is limited. Thus, it is undesirable to use such communication infrastructures for participatory sensing. Meanwhile, the local direct communication functions of portable devices have recently been put to practical use (e.g., LTE Direct, iBeacon). Due to these reasons, Mobile Wireless Sensor Networks (MWSNs), which consist of mobile sensor nodes (hereafter nodes) with local direct communication functions, have recently attracted much attention as a means of achieving participatory sensing with no infrastructure [16]. In MWSNs, nodes typically sends their sensor readings and location information to a sink, which gathers and manages the information. These networks can be well utilized in situations where applications gather sensor data with geographical conditions (e.g., sensor data acquired around Tokyo Station).

In MWSNs constructed by mobile nodes held by ordinary people, the number of nodes is generally very large, and thus, each node has many nodes which can directly communicate with each other (called neighboring nodes). We call such networks dense MWSNs. In these networks, it is desirable to reduce the traffic required for satisfying application requirements as much as possible in order to avoid exhausting the limited wireless bandwidth and energy of nodes.

Most MWSN applications require environmental information, such as temperature or humidity, which is used to detect particular areas (called events) where sensor readings have a higher value than a threshold specified for the broader region. For example, some applications request ar- 
eas (events) where the local temperature exceeds $30^{\circ} \mathrm{C}$.

In order to detect an event, it is effective to estimate the perimeter (or boundary) of the event. Typically, such boundary can be estimated using only the sensor data (location information and sensor readings) of nodes near the boundary $[2,7]$. In other words, it is not necessary to gather sensor data from nodes far from the target boundary. In this context, we have previously proposed a method which efficiently detects nodes near boundaries by utilizing the overhearing of sensor data in dense MWSNs [12]. In this method, nodes determine that they are located near the target boundary when they are adjacent to a node with different sensor reading on a Voronoi diagram. Here, 'different' means that the respective pair of sensor readings are classified into different groups according to the specified threshold; i.e., one is lower than the threshold, and the other higher than or equal to the threshold. Each node determines whether it is located near the target boundary based on a Voronoi diagram constructed using only overheard sensor data.

Typical events requested in MWSNs include regions of sudden rain or air pollution [8], in addition to those with higher (or lower) temperature and humidity. The locations or shapes of such events are expected to change drastically as time passes. Thus, when an application requests the detection of such an event, the sink must rapidly gather sensor data, or the detected boundary will be significantly different from the actual boundary at the time of the request. In these situations, the application typically sets a deadline for data gathering. In such deadline-constrained data gathering, it is known that the number of sensor data which can be gathered becomes limited $[1,6]$. Our previous method, however, does not consider the above limitation and tries to gather sensor data from all detected nodes. Thus, when this method is applied to a deadline-constrained environment, many sensor data may be missed due to frequent packet collisions. This results in the waste of much traffic involved in the transmission of sensor data which ends up failing to be gathered by the sink (i.e., cannot be used for estimating the target boundary). Such wasted traffic should be eliminated in MWSNs with limited channel bandwidth.

To solve this problem, it is necessary to reduce the number of sensor data sent toward the sink. However, if we simply reduce the number of sensor data without due consideration, the accuracy of the estimated boundary may deteriorate. Thus, it is also important to suppress the deterioration in the accuracy of the estimated boundary. To achieve these ends, we propose in this paper a method which utilizes the sampling of nodes (detected as in our previous method), with consideration of the target boundary shape. Our proposed method first takes samples of detected nodes, such that the distance between sampled nodes is less than a specified threshold, and only sampled nodes send their sensor data to the sink. But in addition, our proposed method also additionally gathers (or removes) sensor data from the chosen samples when the accuracy of the estimated boundary is expected to be improved. Note that these procedures are achieved only through the autonomous behavior of nodes responding to the overhearing of sensor data transmitted to the channel. In this way, the number of packet collisions (and thus, the wasted traffic) can be reduced, while suppressing deterioration in the accuracy of the estimated boundary, because the number of sensor data sent toward the sink can be reduced through consideration of the target boundary shape.

The contributions of this paper are summarized as follows:

- Our proposed method can determine target boundary shapes, within a given deadline, with low traffic waste in dense MWSNs. To the best of our knowledge, there are no existing studies on achieving these aims in mobile environments.

- To reduce traffic waste while suppressing deterioration in the accuracy of the estimated boundary, our proposed method introduces the novel approach of sampling nodes with consideration of the target boundary shape.

- Through extensive simulations, we show that our proposed method works well in terms of reducing traffic waste for data gathering.

The remainder of this paper is organized as follows. In Section 2, we describe the assumptions of this paper. In Section 3, we introduce related works. In Section 4, we present our previous method. In Section 5, we detail our proposed method. In Section 6, we show the results of the simulation experiments, and in Section 7, we summarize this paper.

\section{ASSUMPTIONS}

\subsection{Network configuration}

In this paper, we assume a dense MWSN consisting of a large number of mobile nodes, which are held by ordinary people and equipped with a radio communication function. Each node can communicate directly with nodes located in the circle with radius $r$, centered on itself (called the communication area). Each node is equipped with sensor devices which observe (sense) physical phenomena, but does not know the application requirement. Thus, each node performs a sensing operation only once when informed about the requirement by receiving a query (described in Section 4.1). Each node can obtain its own location information at an arbitrary time, using a positioning devices such as GPS. Nodes move freely over the entire region. The sink is stationary and gathers sensor data from the mobile nodes.

\subsection{Application requirements}

The sensing region is assumed to be a two-dimensional plane. We assume that an application seeks a target boundary (the perimeter of a specific event) in the sensing region at a certain time. According to the application requirement, the sink sets a threshold for the sensor reading, and gathers sensor data (a sensor reading and the corresponding location) in order to estimate the boundary.

\subsection{Boundary estimation}

As a mean of estimating the boundary, the sink uses a method based on Delaunay triangulation, where the vertices are node locations. Specifically, the sink constructs Delaunay triangles whose circumcircles contain no vertices, based on the location information of received sensor data. Then, the sink draws line segments joining the midpoints of the sides of the Delaunay triangles (called Delaunay edges) whose endpoint nodes observe different sensor readings from 


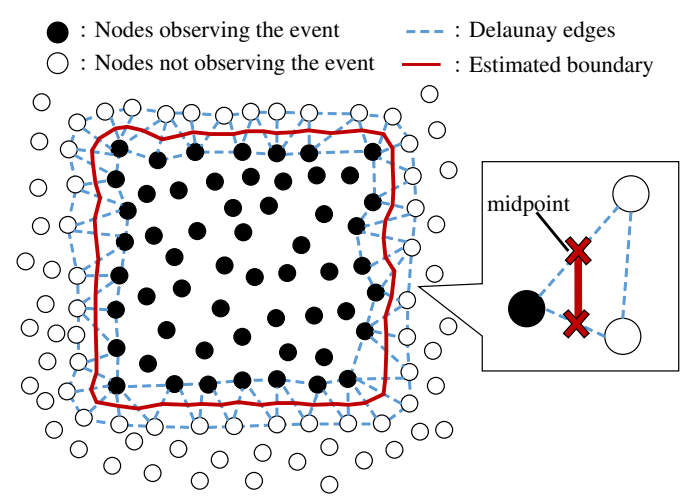

Figure 1: The boundary estimated by the sink.

each other. An example of one such estimated boundary is shown in Figure 1.

The vertices of a Delaunay triangle are located nearer to each other than those of other triangles in such configurations, because a Delaunay triangle has no vertecies inside its circumcircle. Based on these characteristics, nodes can determine that they are located far from the boundary when they are not adjacent to nodes with different sensor readings on the Delaunay diagram; and the sensor readings of such nodes need not be gathered in order to estimate the boundary. In this paper, we call nodes adjacent to a node with a different sensor reading on the Delaunay diagram, boundary nodes. In addition, we call the sequence of procedures that includes the detection of boundary nodes, collection of their sensor data, and estimation of the target boundary, boundary detection.

\section{RELATED WORK}

In $[9,13]$, the authors have proposed methods which reduce the traffic for monitoring boundaries, by taking samples of nodes near the target boundary in environments where nodes are deployed at fixed locations. These methods take nodes samples near the boundary with equal intervals determined based on the communication area. When a sampled node becomes far from the boundary, due to change in the boundary, these methods remove the node from the sample group and take another boundary node sample (instead of the removed node), while maintaining the interval between sampled nodes.

Similarly to our proposed method, these methods take node samples near the target boundary with equal intervals. However, there, the authors assume that the node locations are all fixed, and all nodes recognize all the locations of their neighboring nodes. Thus, if these methods are applied to dense MWSNs, a large amount of traffic will be required for recognizing the locations of neighboring nodes, because node locations change dynamically over time. In addition, these methods do not consider the shape of the target boundary when taking node samples. Thus, the methods may result in significant deterioration in the accuracy of boundary estimation.

\section{OUR PREVIOUS METHOD}

In this section, we describe our previous method proposed in [12]. In this method, the sink propagates a query (Section 4.1) to inform all nodes of the application requirement. After the query propagation process, boundary nodes are detected (Section 4.2), and the sink gathers their sensor data according to the procedure described Section 4.3.

\subsection{Query propagation}

The query propagation process is initiated by the sink. In this process, each node (including the sink) uses a locationbased flooding protocol based on that proposed in [15], to inform all the nodes over the entire sensing region about the requirement, with minimum traffic. The query contains the following information on the application requirement:

- the overall sensing region.

- the type of sensor reading.

- the threshold of the sensor reading for detecting the event.

The query also contains information on the identifier and sensor data of the transmitter of the query. In addition, the query contains (a) the allotted detection time (i.e., the time allotted for the boundary node detection process), $t_{\text {detect }}$, as the information on the condition for terminating the boundary node detection process described in Section 4.2, and (b) the number of hops from the sink, which is used in the data gathering process described in Section 4.3.

First, the sink generates and transmits the query over its communication area. On receiving the query, each node obtains its own sensor reading, as specified by the query, at its current location. After this, it updates the information on the transmitter to its own identifier and sensor data recorded in the query, and re-transmits the updated query over its communication area after a specified waiting time. The waiting time is set shorter as the distance from the transmitter increases. Before the waiting time expires, each node monitors the channel. Upon receiving a query transmitted by a neighboring node, the receiving node treats the product of communication areas of itself and the transmitter as 'covered.' If the product of its own communication area and the sensing region specified by the application is fully covered, before the waiting time has expired, the node stops transmitting the received query.

In addition, each node records the identifier of the transmitter of the received query; and using this information, a tree-shaped network is constructed, whose root is the sink and whose nodes are the transmitters of the query. We call this network the forwarding tree. The forwarding tree is used for the data gathering described in Section 4.3.

Furthermore, each node checks whether its own sensor reading is different from that of the transmitter of the query. If so, it determines that the boundary exists in its communication area and starts the boundary node detection process described in the next section. In this way, excessive exchange of sensor data can be suppressed in areas far from the target boundary. In our previous method, this query propagation technique is effectively utilized for the first phase of the boundary node detection process.

\subsection{Boundary node detection}

This process detects boundary nodes using Voronoi diagrams, which are duality with Delaunay diagrams. Here, nodes send their sensor data only when they determine that 
they are boundary nodes based on Voronoi diagrams constructed using only overheard sensor data. In this way, the traffic required for detecting boundary nodes is suppressed.

We present the details of this process below, assuming the situation depicted in Figure 2, where nodes $\{1, \ldots, 6\}$ receive a query transmitted by node 7 . Here, black and white nodes have different sensor readings, and the grey nodes (with dashed borders) have not sent their sensor data yet. All nodes can communicate directly with each other.

Each node constructs a Voronoi diagram, based on overheard sensor data and its own sensor data every time it overhears the query or sensor data sent in this process. Based on this Voronoi diagram, each node determines whether it is a boundary node until the allotted detection time $\left(t_{\text {detect }}\right)$ elapses according to the following procedures:

- When a node is adjacent to a node with a different sensor reading in the constructed Voronoi diagram, it sets a timer, $T_{\text {boundary }}$, which is set shorter as the distance between itself and the node decreases. When it does not overhear new sensor data until the timer expires, it sends its own sensor data to its parent in the forwarding tree. Figure 2(a) shows the Voronoi diagram constructed by node 1 . Note that node 1 does not yet recognize the existence of nodes $\{2, \ldots, 6\}$, because these nodes have not yet sent their sensor data. In this figure, node 1 is adjacent to node 7 (with a different sensor reading). Thus, node 1 sets a $T_{\text {boundary }}$ based on the distance between itself and node 7 . Node 2 proceeds similarly to node 1 . Node 1 sends its sensor data to node 7 earlier than node 2 , because it is nearer to node 7 than node 2 .

- When a node is not adjacent to a node with a different sensor reading in the Voronoi diagram, it does not send its sensor data. Figure 2(a) shows the Voronoi diagram constructed by node 5 , after node 5 has overheard sensor data from nodes $\{1, \ldots, 4,6\}$. As can be seen, node 5 is not adjacent to nodes with different sensor readings; thus, node 5 does not send its sensor data.

\subsection{Data gathering}

When the allotted detection time, $t_{\text {detect }}$, has elapsed after the query had been generated, each node in the forwarding tree sets a waiting time for data gathering. After this waiting time elapsed, each node puts all the received sensor data into a packet, and sends the packet to its parent. When the parent receives the packet, it sends an ACK to the child that has sent the packet. The child resends the packet until it receives the ACK from its parent. If no ACK has been received after the node has resent the packet for a predetermined time, the node stops sending the packet. When a node receives a packet from its child after sending its own packet, it immediately forwards the received packet to its parent. Each node sets its waiting time such that the value decreases with an increase in the number of hops from the sink. In this way, nodes in the forwarding tree send their packets in descending order of number of hops from the sink. After all nodes in the forwarding tree have sent their packets, the sink can gather all the sensor data sent during the boundary node detection process.

\subsection{Problems in our previous method}

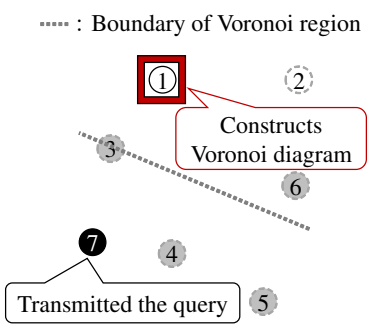

(a) site points: nodes 1 and 7 .

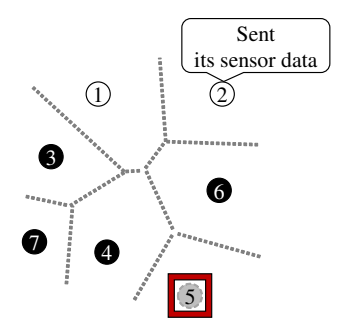

(b) site points: nodes $\{1, \ldots, 7\}$.
Figure 2: An example of boundary node detection.

Our previous method does not enable deadline-constrained data gathering. Specifically, nodes must set longer waiting times for data gathering in order for all the sensor data sent in the process described in Section 4.2 to be gathered, while avoiding packet collisions. And when the method attempts to gather sensor data from all boundary nodes by a specified deadline, through reducing the waiting time, many sensor data may be missed due to frequent packet collisions. This typically results in the waste of much traffic, due to the transmission of sensor data that ends up failing to be gathered by the sink (i.e., cannot be used for estimating the target boundary).

\section{OUR PROPOSED METHOD}

In this section, we describe the application requirement, outline and design policy, and detailed behavior of the proposed method.

For the purposes of explanation, we call the boundary determined by obtaining sensor readings at arbitrary points in the overall sensing region, the ideal boundary; and define the average of the size of areas encompassed by the ideal and estimated boundaries, per meter, as the estimation error.

\subsection{Application requirement}

In addition to the requirements described in Section 2.2, the application sets a deadline, $t_{\text {end }}$, which is the overall operation time permitted after the query is generated, and requires that all sensor data be gathered by the deadline.

\subsection{Design policy}

In order to solve the problems described in Section 4.4, it is necessary to reduce the number of sensor data sent toward the sink while suppressing the increase in the estimation error. One can imagine a naive method to reduce the number of sensor data, which takes samples of detected boundary nodes such that the distance between sampled nodes is less than a specified threshold, as shown in Figure 3(a). When only sampled nodes send their sensor data to the sink, the sensor data can be gathered by the sink in less time, with less packet collisions. However, as shown by the areas surrounded by dotted lines in Figure 3(a), the estimation error may become large when boundary nodes are sampled only on the basis of the distance between sampled nodes. 


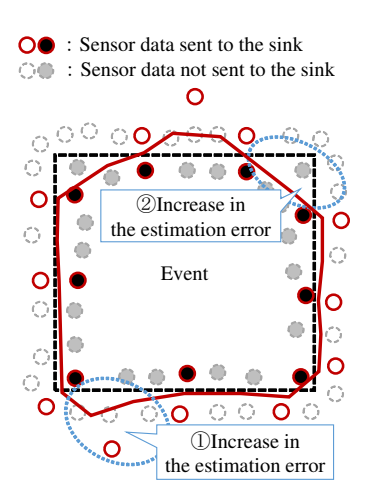

(a) Distance-based sampling

\section{---* : Ideal boundary \\ - : Estimated boundary}

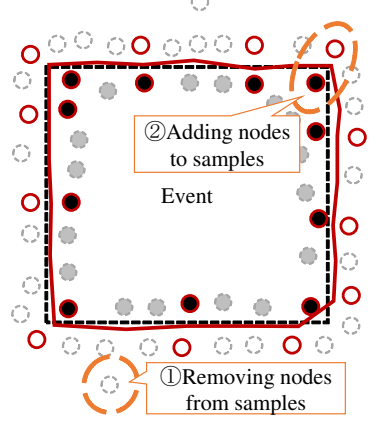

(b) Boundary shapebased sampling
Figure 3: Taking samples of boundary nodes in our proposed method.

To avoid such an increase in the estimation error, our proposed method additionally gathers (or removes) sensor data from the sampled nodes, according to the shape of the ideal boundary, as shown in Figure 3(b). In this way, the number of sensor data sent toward the sink can be reduced, while suppressing the increase in estimation error.

\subsection{Outline of the procedures}

In our proposed method, the sink first propagates the query in the same manner as in our previous method, but here including a deadline for ending the entire boundary detection procedure, $t_{\text {end }}$, in addition to the information described in Section 4.1. After the query is propagated, our proposed method detects and taking samples of boundary nodes, according to the shape of the ideal boundary as described in Sections 5.4 and 5.5; after which, sensor data are gathered by the deadline, as described in Section 5.6.

\subsection{Distance-based sampling}

The method of boundary node detection is the same as described in Section 4.2. However, our proposed method also takes samples of boundary nodes such that the distance between sampled nodes is less than a specified threshold, $l(\leq$ $r)$, to reduce the number of sensor data sent toward the sink. Specifically, a boundary node stops sending its sensor data when it overhears sensor data from another boundary node with the same sensor reading located within the circular area with radius $l$, centered on itself. Figure 4 shows an example of this procedure. Here, sensor data have been sent from nodes $\{1,3,6\}$. After overhearing this sensor data, node 4 stops sending its own sensor data, even though it is adjacent to node 1 and has a different sensor reading on the Voronoi diagram, because nodes 3 and 6 (located within the circular area with radius $l$, centered on node 4 ) have already sent their sensor data with the same sensor readings.

In this way, the number of sensor data sent toward the sink can be reduced. However, two problems may arise:

1. As shown in Figure 5(a), non-boundary nodes (i.e.,

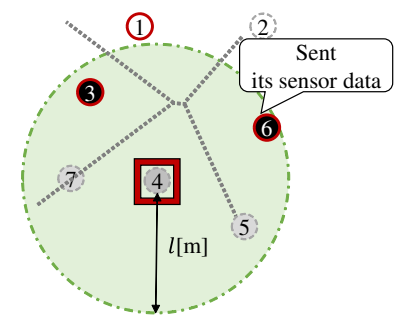

Figure 4: An example of distance-based sampling.

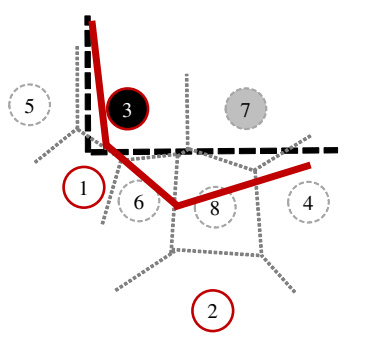

(a) Taking samples of non-boundary nodes.

Figure 5: Examples of the increase in the error of the estimated boundary due to distance-based sampling.

nodes far from the ideal boundary like node 2) may incorrectly determine that they are boundary nodes and send their sensor data. This is because actual boundary nodes, like nodes 6 and 8 , stop sending their sensor data in the above distance-based sampling process. When the sink estimates the boundary using sensor data from nodes (like node 2) far from the ideal boundary, the estimated boundary deviates in the direction of these distant nodes, and this results in an increase in the estimation error. In addition, an actual boundary node, like node 4 , stops sending its sensor data due to the reception of sensor data from node 2 , although the distance between nodes 1 and 4 is longer than $l$.

2. As shown in Figure 5(b), some boundary nodes, like node 5, are not sampled even when their own sensor readings become inconsistent with the estimated boundary; and when the sink estimates the boundary without the sensor data of these boundary nodes, the estimation error increases.

\subsection{Boundary shape-based sampling}

To solve the above problems, each node estimates part of the boundary using overheard sensor data, until it sends its sensor data or the allotted detection time, $t_{\text {detect }}$, has elapsed. Based on this partially estimated boundary, our proposed method removes from the sample-node group, nodes with a high possibility of being non-boundary nodes, and 


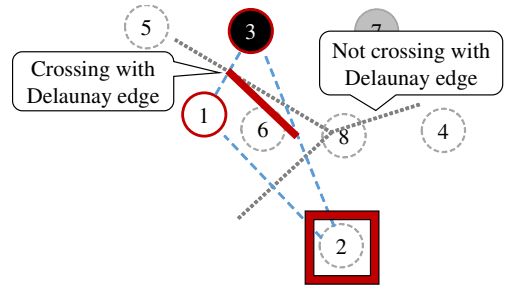

Figure 6: Removing nodes from the sample-node group.

adds to this group, nodes with a high possibility of being boundary nodes. The procedures below are followed by nodes which determine that they are boundary nodes and do not send their sensor data.

\subsubsection{Removing nodes from the sample-node group}

To solve the first problem described in Section 5.4, each node must determine whether is in fact a boundary node, based only on overheard sensor data. Here, a node has the possibility of being a non-boundary node when the distance between itself and the partially estimated boundary is greater than that of other nodes which have already sent their sensor data.

To calculate such relative differences in distance from the estimated boundary, our proposed method exploits the relationship between the relevant Voronoi diagram and Delaunay diagram. The boundary between a pair of adjacent Voronoi regions (called the Voronoi boundary) is the perpendicular bisector of the Delaunay edge whose endpoints are the site points of the regions. When these site points are close to each other, the corresponding Voronoi boundary tends to cross the Delaunay edge. For example in Figure 6 , the Delaunay edge between nodes 1 and 3, located near each other, crosses the corresponding Voronoi boundary. On the other hand, the Delaunay edge between nodes 2 and 3 does not cross the corresponding Voronoi boundary. As this figure suggests, Delaunay edges between nodes located far from each other rarely cross the corresponding Voronoi boundary. In this case, the partially estimated boundary is not adjacent to the respective Voronoi region; e.g., node 2 in Figure 6. This indicates that the distance between that node and the estimated boundary is relatively longer than that of other nodes.

By exploiting this characteristic, our proposed method removes from the sample-node group, nodes with a high possibility of being non-boundary nodes. Specifically, when the partially estimated boundary is not adjacent to a node's own Voronoi region, the node determines that may not be an actual boundary node, and thus, stops sending its own sensor data.

\subsubsection{Adding nodes to the sample-node group}

To solve the second problem described in Section 5.4, each boundary node not sampled in the distance-based sampling process, derives a partially estimated boundary using overheard sensor data. For example, in Figure 7, after node 5 overhears sensor data from nodes $\{1, \ldots, 4\}$, it derives a partially estimated boundary; without, however, using its own sensor data, because it has not been sampled at this time. After this, the node checks whether its own sensor

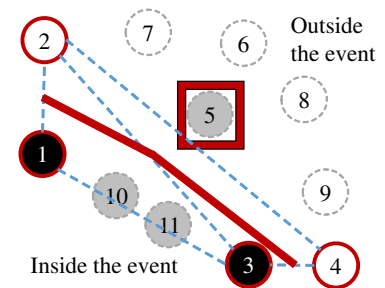

Figure 7: Adding nodes to the sample-node group.

data is inconsistent with the derived boundary. If so, it sets a timer, $T_{m o d}$, for sending this sensor data (called mod-data) to modify the estimated boundary. $T_{m o d}$ is set shorter as the distance between the node and the partially estimated boundary increases. If the node receives no sensor data with the same sensor reading as its own, from a node located within the circular area with the radius $l$, centered on itself, before the timer elapses, it sends the mod-data to its parent in the forwarding tree. This means that the mod-data is added to the sample-node group.

To appropriately modify the estimated boundary using such mod-data, it is necessary to gather sensor data from the nearest boundary node with a different sensor reading from the sender of the mod-data. To achieve this, when a boundary node receives such mod-data and has a different sensor reading from that in the mod-data, it sets a timer, $T_{\text {pair-mod }}$, for sending its own sensor data, which is set shorter as the distance between itself and the sender node decreases. If it has already set $T_{\text {boundary }}$, it cancels $T_{\text {boundary }}$ and instead waits to send its sensor data according to the newly set $T_{\text {pair-mod }}$. If it does not receive sensor data from a node with the same sensor reading located within the circular area with the radius $l$, centered on itself, before the timer elapses, it sends its sensor data to its parent in the forwarding tree. For example, in Figure 7 , after nodes $\{6, \ldots, 9\}$ receive the mod-data from node 5 , they set $T_{\text {pair }-\bmod \mathrm{S} \text { ac- }}$ cording to the respective distances between themselves and node 5. Among nodes $\{6, \ldots, 9\}$, node 6 sends its sensor data earlier than the others because it is the nearest to node 5 .

\subsection{Data gathering}

In the processes described in Sections 5.4 and 5.5, sensor data is sent no later than the time when the allotted detection time, $t_{\text {detect }}$, has elapsed after the query had been generated. In order for the sink to receive this sensor data by the deadline, $t_{\text {end }}$, it is necessary to complete the data gathering within a specified period $\left(t_{\text {end }}-t_{\text {detect }}\right)$. To achieve this, each node in the forwarding tree sends sensor data received from its child to its parent after the following time, $W^{g}$, passes:

$$
W^{g}=\frac{t_{\text {end }}-t_{\text {detect }}}{h_{\max }}-\text { delay }_{\text {trans }}+\text { rand } .
$$

In the above equation, $h_{\max }$ is the maximum number of hops from the sink, which is determined by the location of the sink, the size of the sensing region and the communication range. delaytrans is the transmission delay per hop. rand is a random number within a certain range, to reduce packet collisions. The first right-side term of Eq.(1) shows the time available to each node for receiving and sending sensor data, 


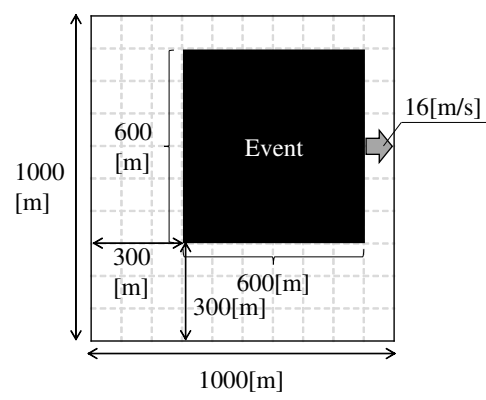

Figure 8: The event used in the simulation.

per hop. By subtracting delaytrans from this term, each node can calculate the time available for forwarding received sensor data.

Each time a node receives sensor data from its child, it forwards this data after $W^{g}$ has elapsed from the time of its reception. In addition, no nodes resend the same sensor data, to avoid increased traffic waste due to packet collisions. In this way, the sink can receive by the deadline, $t_{\text {end }}$, the requisite sensor data sent by the allotted detection time, $t_{\text {detect }}$.

\section{PERFORMANCE EVALUATION}

In this section, we show the results of simulation experiments evaluating the performance of our proposed method. For the simulations, we used the network simulator, Scenargie 2.0 [14].

\subsection{Simulation Model}

There are $N$ mobile nodes and a sink in a two-dimensional sensing region of $1,000[\mathrm{~m}] \times 1,000[\mathrm{~m}]$. The sink is fixed at the point $(0[\mathrm{~m}], 0[\mathrm{~m}])$ from the left and bottom edges of the sensing region. Nodes move according to the random waypoint mobility model [3]. Specifically, each node randomly selects its destination in the sensing region. After determining their destination, nodes move there at a constant speed, uniformly determined within the range of $[0.5$, $1.0][\mathrm{m} / \mathrm{s}]$. On arriving at their destination, nodes stop there for $60[\mathrm{~s}]$ before determining their next destination. The sink and nodes communicate with IEEE $802.11 \mathrm{~g}$, with a communication range, $r$, of roughly $100[\mathrm{~m}]$. The allotted detection time, $t_{\text {detect }}$, is set to $0.3[\mathrm{~s}]$ after query generation.

Figure 8 shows the event used in the simulation. For the experiments, we adopted a square-shaped event of $600[\mathrm{~m}] \times 600[\mathrm{~m}]$ which begins at the position shown in Figure 8, and then moves to the right, as shown by the arrow, at $16[\mathrm{~m} / \mathrm{s}]$.

\subsection{Evaluation criteria}

In the simulation experiment, we evaluated the following three criteria:

- Total traffic is defined as the sum of the size of all packets sent by the sink and nodes, at the application layer. Table 1 shows the size of each message.

- Wasted traffic is defined as the sum of the traffic generated in sending sensor data that failed to be received by the sink.
Table 1: Message size.

\begin{tabular}{c|l|l|l}
\hline Method & \multicolumn{1}{|c|}{ Roll } & Message name & Size[B] \\
\hline \hline Proposed & $\begin{array}{l}\text { Boundary nodes } \\
\text { detection \& sampling }\end{array}$ & Sensor data & $21+24$ \\
\hline $\begin{array}{c}\text { Previous \& } \\
\text { Distance-based sampling }\end{array}$ & $\begin{array}{l}\text { Boundary nodes } \\
\text { detection \& sampling }\end{array}$ & Sensor data & $20+24$ \\
\hline Common & $\begin{array}{l}\text { Query propagation } \\
\text { Data gathering }\end{array}$ & $\begin{array}{l}\text { Query } \\
\text { Data packet }\end{array}$ & $\begin{array}{l}92 \\
26+24\end{array}$ \\
\hline
\end{tabular}

- Estimation error is defined as the average of the areas encompassed by the ideal and estimated boundaries, per meter. This value increases as the difference between the ideal and estimated boundaries increases. In addition, in order to verify the effectiveness of the boundary estimated by each method, we separately measured the estimation error resulting from using sensor data of all nodes in the sensing region (i.e., ideal error). Here, even if the sink can gather sensor data from all nodes without packet collisions, it cannot estimate the ideal boundary (i.e., the ideal error is $0[\mathrm{~m}])$. This is because the set of acquired data is geographically discrete.

\subsection{Methods evaluated}

Our proposed method is designed to reduce traffic waste while suppressing estimation error, by implementing the following two approaches:

- taking samples of boundary nodes based on the distance between nodes.

- removing from the sample-node group, nodes with a high possibility of being non-boundary nodes; and adding to the group, nodes with a high possibility of being boundary nodes; according to the shape of the ideal target boundary.

In order to verify the effectiveness of each of these approaches, we investigated the performance of the following three methods:

- Proposed method is defined as the method described in Section 5. The value of $l$ used in the distance-based sampling process is set at $50[\mathrm{~m}]$.

- Previous method is defined as the method described in Section 4. In order to gather all sensor data by the deadline, this method gathers sensor data according to the process described in Section 5.6.

- Distance-based sampling method is defined as the proposed method without the boundary shape-based sampling process described in Section 5.5. The value of $l$ is set at $45[\mathrm{~m}]$, which results in roughly the same traffic as in the proposed method when the number of nodes is 1,000 according to preliminary experiments.

\subsection{Impact of $t_{\text {end }}$}

The traffic waste depends on the deadline. Sensor data are more likely to collide with others when the sending times are almost the same. Such collisions occur frequently when the allotted time is short. In this experiment, we varied the value of $t_{\text {end }}$, the allotted operation time after the query is generated, in order to confirm the impact of the deadline. 


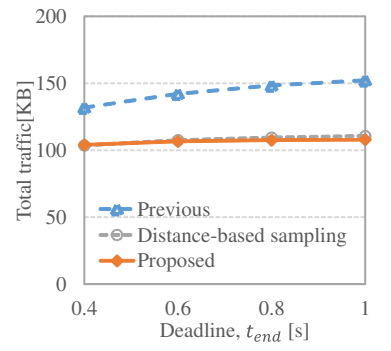

(a) Total traffic.

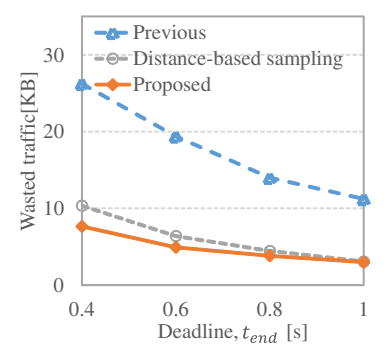

(b) Wasted traffic.

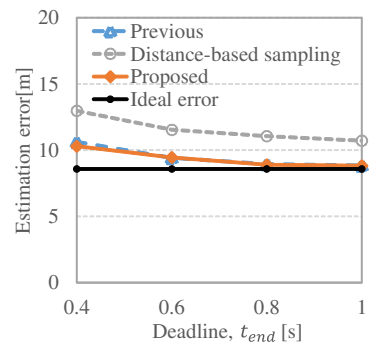

(c) Estimation error.

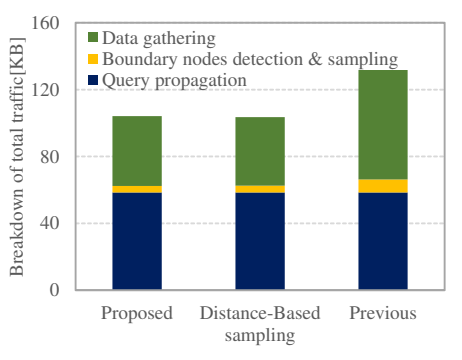

Figure 10: Breakdown of total traffic.

Figure 9: Impact of $t_{\text {end }}$.

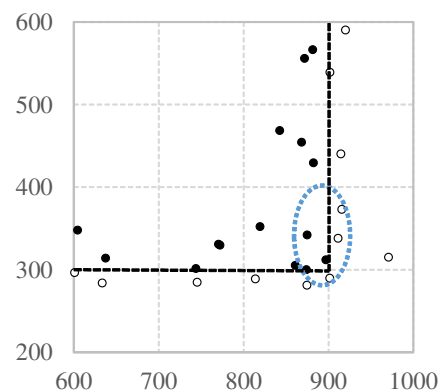

(a) Proposed method.

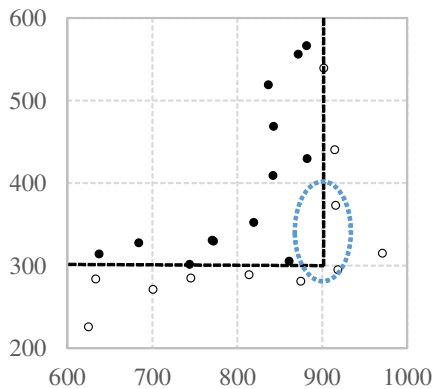

(b) Distance-based sampling method.

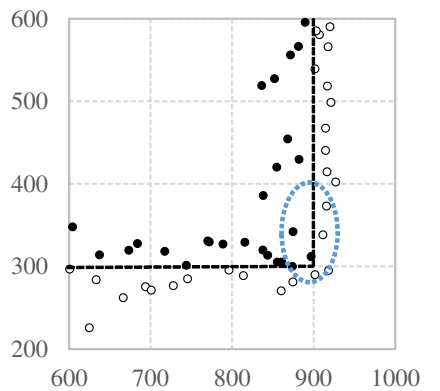

(c) Previous method.

Figure 11: The distribution of nodes which have sent their sensor data.

Figure 9 shows the results with varying $t_{e n d}$, when $N$ is 1,000. Figures 9(a), 9(b), and 9(c) show the total traffic, wasted traffic, and estimation error, respectively. The horizontal axis of these graphs indicates the deadline, $t_{\text {end }}$.

From Figure 9(a), we can see that the total traffic in each method increases as $t_{\text {end }}$ increases. This is because the frequency of packet collisions decreases as the time available for data gathering increases. When the packet collisions decrease, more sensor data are correctly gathered by the sink. In addition, we can see that the total traffic in the previous method is greater than in the proposed and distance-based sampling methods. This is because the latter two methods reduce the number of sensor data sent toward the sink by taking samples of boundary nodes. To confirm this, we separately measured the breakdown of the total traffic when $t_{\text {end }}$ was $0.4[\mathrm{~s}]$. Figure 10 shows the results. We can see that the proposed and distance-based sampling methods clearly reduce the traffic required for boundary node detection and sampling, compared with the previous method. This means that the traffic required for data gathering in the proposed and distance-based sampling methods is less than in the previous method.

From Figure 9(b), we can see that the wasted traffic in each method decreases as $t_{\text {end }}$ increases. This is due to the decrease in packet collisions. In addition, we can see that the respective traffic waste in the proposed and distancebased sampling methods is less than in the previous method. The former methods reduce the number of sensor data sent toward the sink, by taking samples of boundary nodes, and this leads to fewer packet collisions than in the previous method.

From Figure 9(c), we can see that the estimation error in each method decreases and approaches the ideal error (about $8[\mathrm{~m}]$ in this simulation environment) as $t_{\text {end }}$ increases. This is because the number of sensor data received by the sink increases. We can also see that the estimation error in the distance-based sampling method is higher than in the previous method. This is due to the problems described in Section 5.4; unlike the distance-based sampling method, the proposed method can suppress the increase in the estimation error, by adding and removing sensor data, according to the shape of the ideal target boundary.

In order to investigate the difference in estimation error among the methods in Figure 9(c), we plotted (in Figure 11) the geographical distribution of nodes which have sent their own sensor data (called senders in this section) during the boundary node detection and sampling processes, in each method, when $t_{\text {end }}$ was $0.4[\mathrm{~s}]$. In addition, in Figure 12, we 


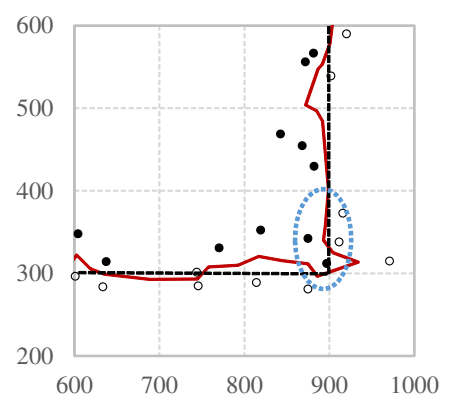

(a) Proposed method.

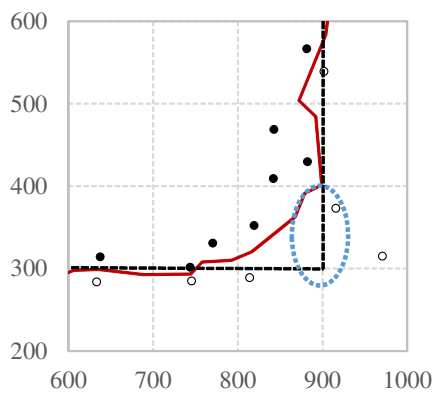

(b) Distance-based sampling method.

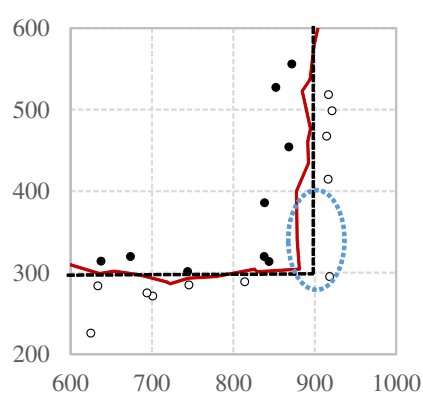

(c) Previous method.

Figure 12: The distribution of nodes whose sensor data can be successfully received by the sink and the estimated boundary.

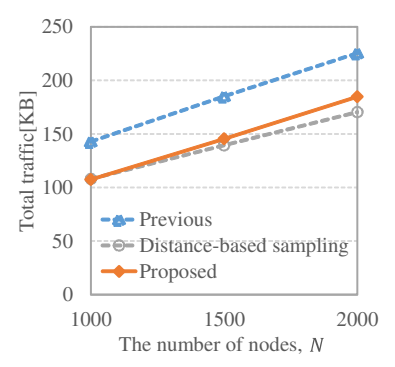

(a) Total traffic.

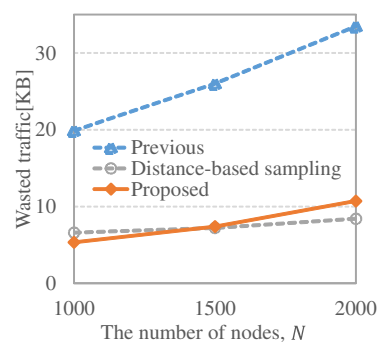

(b) Wasted traffic.

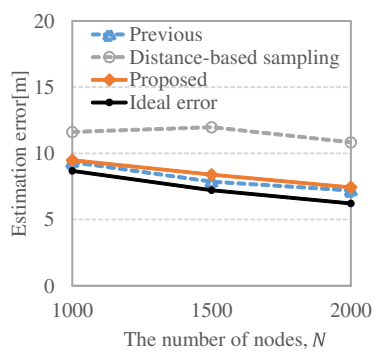

(c) Estimation error.

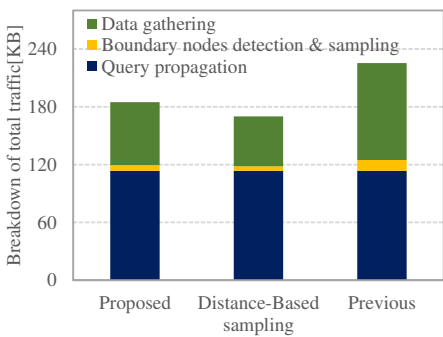

Figure 14: Breakdown of total traffic.

Figure 13: Impact of $N$.

show the geographical distribution of nodes whose sensor data have been successfully received by the sink and the estimated boundary, in each method, in the same situation as Figure 11. From Figure 11(b), we can see that the distancebased sampling method does not take samples of some nodes in the area encompassed by the dotted line. Thus, in the distance-based sampling method, the estimated boundary encompassed by this line in Figure 12(b) deviates from the ideal boundary. In contrast, as we see in Figure 11(a), the proposed method takes samples of these nodes, and thus the estimated boundary encompassed by the dotted line in Figure 12(a) approaches the ideal boundary. From Figures 11(c) and 12(c), we can see that many sensor data in the area encompassed by the dotted line are missed in the previous method. However, the sink can estimate the boundary near the ideal boundary because it happens to receive one sensor data from the corner of the event.

\subsection{Impact of $N$}

The total traffic depends on the number of boundary nodes, which varies with the total number of nodes in the sensing region, and with the length of the boundary. In this experiment, we varied the value of $N$ in order to confirm the impacts of the number of boundary nodes.
Figure 13 shows the results with varying $N$, when $t_{\text {end }}$ is $0.6[\mathrm{~s}]$. Figures 13(a), 13(b), and 13(c) show the total traffic, wasted traffic, and estimation error, respectively. The horizontal axis of these graphs indicates the number of nodes, $N$.

From Figure 13(a), we can see that the total traffic in each method increases as $N$ increases. This is due to the increase in the number of boundary nodes. In addition, we can see that the total traffic in the proposed method is greater than in the distance-based sampling method, when $N$ is larger than 1,000. This is because, in the former method, the number of mod-data transmissions increases as $N$ increases. To confirm this, we separately measured the breakdown of the total traffic when $N$ was 2,000. Figure 14 shows the results. We can see that the traffic required for data gathering in the proposed method is clearly greater than in the distance-based sampling method. This reflects the greater number of data sent during the boundary shape-based sampling process (Section 5.5), because the distance-based sampling method does not implement the boundary shape-based sampling process.

From Figure 13(b), we can see that the wasted traffic in each method increases as $N$ increases. This is because the 
frequency of packet collisions increases as the number of boundary nodes increases. In addition, we can see that the respective traffic waste in the proposed and distance-based sampling methods is less than in the previous method. This is due to the same reasons as described in Section 6.4. We can also see that, when $N$ is 2,000 , the wasted traffic in the proposed method is greater than in the distance-based sampling method. This is due to the increase in the number of mod-data transmissions, as described above.

From Figure 13(c), we can see that the estimation error in each method decreases as $N$ increases. This is because, when $N$ increases, the overall number of sensor data received by the sink increases, though the frequency of packet collisions also increases. In addition, we can see that the estimation error in the distance-based sampling method is higher than in the previous method, while the increase in the estimation error in the proposed method can be suppressed. This is due to the same reasons as described in Section 6.4.

\section{CONCLUSION}

In this paper, we have proposed a method for dense MWSNs, which reduces the number of sensor data sent toward the sink, while suppressing the increase in estimation error, by taking samples of boundary nodes with consideration of the shape of the ideal target boundary. Specifically, our proposed method takes samples of boundary nodes such that the distance between sampled nodes is less than a specified threshold; and suppresses the increase in the estimation error by removing from the sample-node group nodes with a high possibility of being non-boundary nodes, and adding to the group nodes with a high possibility of being boundary nodes, when the estimation error is expected to be improved. To achieve this, each node determines whether it should (or should not) send its own sensor data based on the partially estimated boundary derived from overheard sensor data. In this way, traffic waste can be reduced, while the increase in the estimation error is suppressed. The results of the simulation experiments confirm that our proposed method can achieve these objectives.

In this paper, we assume that the application seeks the target boundary at the time when the query is generated. However, some applications require real-time monitoring of the changes in the target boundary $[9,17]$. In such real-time monitoring, it is important to gather sensor data in a short period. Thus, as part of our future work, we plan to propose a method, which can monitor the boundary efficiently in real time, in dense and mobile environments, based on the method proposed here.

\section{Acknowledgment}

This research is partially supported by JSPS Fellows (JP16J05362) and the Grant-in-Aid for Scientific Research (A)(26240013) of the Ministry of Education, Culture, Sports, Science and Technology, Japan, and JST, Strategic International Collaborative Research Program, SICORP, and the Cooperative Research Project of the RIEC, Tohoku University.

\section{REFERENCES}

[1] B. Alinia, M. H Hajiesmaili and A. Khonsari, "On The Construction of Maximum-Quality Aggregation Trees in Deadline-Constrained WSNs," Proc. INFOCOM, pp.226-234, 2015.
[2] B. Avci, G. Trajcevski and P. Scheuermann, "Managing Evolving Shapes in Sensor Networks," Proc. SSDBM, 2014.

[3] T. Camp, J. Boleng and V. Davies, "A Survey of Mobility Models for Ad Hoc Networks Research," Wireless Communications and Mobile Computing, Vol.2, No.5, pp.483-502, 2002.

[4] D. Estrin, "Participatory sensing: applications and architecture," IEEE Internet Computing, Vol.14, No.1, pp.12-42, 2010.

[5] Global Mobile Data Traffic Forecast Update, 2015-2020. In Cisco White Paper, 2016.

[6] S. Hariharan and N. B. Shroff, "Maximizing Aggregated Information in Sensor Networks Under Deadline Constraints," IEEE Trans. Automatic Control, Vol.56, No.10, pp.2369-2380, 2011.

[7] M. I. Ham and M. A. Rodriguez, "A Boundary Approximation Algorithm for Distributed Sensor Networks," Int. Journal of Sensor Networks, Vol.8, No.1, pp.41-46, 2010.

[8] D. Hasenfratz, O. Saukh, S. Sturzenegger and L. Thiele, "Participatory Air Pollution Monitoring Using Smartphones," Mobile Sensing, pp.1-5, 2012.

[9] G. Jin and S. Nittel, "Efficient Tracking of 2D Objects with Spatiotemporal Properties in Wireless Sensor Networks," Distributed and Parallel Databases, Vol.29, No.1-2, pp.3-30, 2011.

[10] W. Z. Khan, Y. Xiang, M. Aalsalem and Q. Arshad, "Mobile Phone Sensing Systems: A Survey," IEEE Communications Surveys \& Tutorials, Vol.15, No.1, pp.402-427, 2013.

[11] N. D. Lane, E. Miluzzo, H. Lu, D. Peebles, T. Choudhury and A. T. Campbell, "A Survey of Mobile Phone Sensing," Communications Magazine, Vol.48, No.9, pp.140-150, 2010.

[12] K. Matsuo, K. Goto, A. Kanzaki, T. Hara and S. Nishio, "Overhearing-Based Efficient Boundary Detection in Dense Mobile Wireless Sensor Networks," Proc. MDM, 2014.

[13] S. Park, S. Hong, E. Lee, S. Kim and N. Crespi, "Large-Scale Mobile Phenomena Monitoring with Energy-Efficiency in Wireless Sensor Networks," Computer Networks, Vol.81, pp.116-135, 2015.

[14] Scenargie2.0 Base Simulator revision 18021, Space-Time Engineering, November 2015. [Online]. Available: https://www.spacetime-eng.com/

[15] Y. Tseng, S. Ni, Y. Chen and J. Sheu, "The Broadcast Storm Problem in a Mobile Ad Hoc Network," Wireless Networks, Vol.8, No.2-3, pp.153-167, 2002.

[16] G. S. Tuncay, G. Benincasa and A. Helmy, "Autonomous and Distributed Recruitment and Data Collection Framework for Opportunistic Sensing," ACM SIGMOBILE Mobile Computing and Communications Review, Vol.16, No.4, pp.50-53, 2012.

[17] X. Zhu, R. Sarkar, J. Gao and J. S. Mitchell, "Light-Weight Contour Tracking in Wireless Sensor Networks," Proc. INFOCOM, pp.1175-1183, 2008. 